A. Kodama

Nagoya Math. J.

Vol. 73 (1979), 1-5

\title{
ON BIMEROMORPHIC AUTOMORPHISMS OF HYPERBOLIC COMPLEX SPACES
}

\author{
AKIO KODAMA
}

\section{Introduction}

Let $X$ be a hyperbolic complex space ${ }^{(1)}$ in the sense of S. Kobayashi [2]. We write $\operatorname{Aut}(X)$ (resp. $\operatorname{Bim}(X))$ for the group of all biholomorphic (resp. bimeromorphic) automorphisms of $X$.

In this note, we shall prove

THEOREM 1. Let $f$ be a meromorphic mapping from a complex manifold $M$ into a hyperbolic complex space $Y$. Then $f$ is holomorphic. In particular, we have $\operatorname{Aut}(X)=\operatorname{Bim}(X)$ for any hyperbolic complex manifold $X$.

In general we have $\operatorname{Aut}(X) \neq \operatorname{Bim}(X)$ for a hyperbolic complex space $X$ with singularities. In fact, we shall show the following

THEOREM 2. There exists a normal irreducible complete hyperbolic complex space $X$ with $\operatorname{Aut}(X) \neq \operatorname{Bim}(X)$.

Thus we have obtained a negative answer to Problem E. 5. in [3].

The author wishes to express his hearty thanks to Professor S. Kobayashi who suggested to prove Theorem 1, and also to Professor T. Ochiai for his help.

\section{Preliminaries}

For later purpose, in this section we shall recall some definitions. A meromorphic mapping $f$ from a complex space $X$ into a complex space $Y$ in the sense of Remmert is a set-valued function satisfying the

Received March 15, 1977.

(1) In this note, by a complex space we mean a reduced irreducible Hausdorff complex analytic space. 
following conditions :

(i) the restriction $\left.f\right|_{W}: W \rightarrow Y$ is a holomorphic mapping for some open dense subset $W$ of $X$;

(ii) the graph $\Gamma_{f}:=\{(x, y) \in X \times Y \mid y \in f(x)\}$ of $f$ is an analytic subset of $X \times Y$ which coincides with the topological closure of the set $\{(x, f(x)) \in X \times Y \mid x \in W\}$ in $X \times Y$;

(iii) the canonical projection $\pi: \Gamma_{f} \rightarrow X$ is proper.

We remark here that the set $W$ in (i) can be chosen in such a way that $X-W$ is an analytic subset of $X$. Let $X, Y$ and $Z$ be three complex spaces. Then, for given meromorphic mappings $f: X \rightarrow Y$ and $g: Y \rightarrow Z$, we can define the composed meromorphic mapping $g \circ f: X \rightarrow Z$ if the full inverse image of $W$ by $f$ is dense in $X$, where $W$ is an open dense subset of $Y$ on which $g$ is holomorphic (cf. Whitney [5]). In general we have $g(f(A)) \neq(g \circ f)(A)$ for a subset $A$ of $X$. We say that $X$ and $Y$ are bimeromorphically (resp. biholomorphically) equivalent if there exist meromorphic (resp. holomorphic) mappings $f: X \rightarrow Y$ and $g: Y \rightarrow X$ such that $g \circ f=\mathrm{id}_{X}$ and $f \circ g=\mathrm{id}_{Y}$. In this case, we call $f$ and $g$ bimeromorphic (resp. biholomorphic) mappings and the inverse to each other. Moreover, in the case of $X=Y$ these are called bimeromorphic (resp. biholomorphic) automorphisms of $X$. A surjective holomorphic mapping $\pi: X \rightarrow Y$ is called a proper modification of $Y$ with center $S$ if it is proper and the restriction $\pi: X-\pi^{-1}(S) \rightarrow Y-S$ is a biholomorphic mapping for some nowhere dense analytic subset $S$ of $Y$. For any proper modification $\pi: X \rightarrow Y$ with center $S$, its inverse is always meromorphic. More precisely speaking, we can define a meromorphic mapping $\psi: Y \rightarrow X$ by using the holomorphic mapping $\pi^{-1}: Y-S \rightarrow X$ $-\pi^{-1}(S)$ and it is the inverse of $\pi: X \rightarrow Y$.

\section{Proof of Theorem 1}

As remarked in Preliminaries, there exists an analytic subset $A$ of $M$ such that the restriction $\left.f\right|_{M_{-A}}: M-A \rightarrow Y$ is holomorphic. Putting $g=\left.f\right|_{M-A}$, we shall prove that $g$ can be extended to a holomorphic mapping.

First we may assume that $A$ is a non-singular complex submanifold of $M$ by the same arguments as in Theorem 4.1, Chap. VI of [2]. Then, since the problem is local, we may further assume that $M$ is a polydisc : 


$$
D \times D^{m-1}=\left\{\left(z, t^{1}, \cdots, t^{m-1}\right) \in C^{m}|| z|<1,| t^{i} \mid<1(1 \leqq i \leqq m-1)\right\}
$$

and $A$ is contained in the subset defined by $z=0$.

For each fixed $t \in D^{m-1}$, we define a holomorphic mapping $g_{t}$ from the punctured disc $D^{*}$ into $Y$ by $g_{t}(z)=g(z, t)$. Once it is shown that $g_{t}: D^{*} \rightarrow Y$ can be extended to a holomorphic mapping $\tilde{g}_{t}: D \rightarrow Y$ for each $t \in D^{m-1}$, the rest of our proof can be done with exactly the same arguments as in Theorem 4.1., Chap. VI of [2]. Thus we have only to show that $g_{t}$ is extendable. By a result of Kwack [4], it is enough to show the existence of a sequence of points $z_{k}$ of $D^{*}$ converging to the origin such that $g_{t}\left(z_{k}\right)$ converges to a point $p$ of $Y$. Now, since $f: M$ $=D \times D^{m-1} \rightarrow Y$ is meromorphic, $f(0, t)$ is a compact analytic subset of $Y$. We take a point $p$ of $f(0, t)$ arbitrarily. Then, by (ii) in section 1 , there are points $z_{k}$ of $D^{*}$ such that the sequence $\left\{\left(\left(z_{k}, t\right), f\left(z_{k}, t\right)\right)\right\}$ converges to the point $((0, t), p)$ of the graph $\Gamma_{f}$ of $f$ in $M \times Y$, because the restriction $\left.f\right|_{D \times\{t\}}: D \times\{t\} \rightarrow Y$ is also meromorphic (cf. [5], Corollary 4. H., p. 196). This implies that $\lim _{k \rightarrow \infty} z_{k}=0$ and $\lim _{k \rightarrow \infty} g_{t}\left(z_{k}\right)=p \in Y$, and hence the proof is completed.

q.e.d.

LEMMA 1. There exist a normal irreducible complex space $S, a$ compact hyperbolic complex manifold $T$ and a meromorphic mapping $f: S \rightarrow T$ which is not holomorphic.

Proof. Let $T$ be a compact projective algebraic manifold which is hyperbolic. Then, as remarked in [2], p. 100, $T$ can be imbedded into some complex projective space $P_{n}(C)$ in such a way that $T$ is projectively normal, that is, the affine cone $C(T):=$ all complex lines through the origin $o$ of $C^{n+1}$ representing the points of $T$ \} is a normal complex space. It is clear that $C(T)$ is non-singular except at the origin. Let $\pi: C(T)-\{o\} \rightarrow T$ be the restriction of the natural projection $C^{n+1}-\{o\}$ $\rightarrow P_{n}(C)$. Then, obviously $\pi$ cannot be extended to a holomorphic mapping from $C(T)$ into $T$. On the other hand, by the theorem of resolution of singularities by Hironaka [1] and an extention theorem by Kwack [4] it is easily verified that $\pi$ can be extended to a meromorphic mapping $\tilde{\pi}: C(T) \rightarrow T$. The triple system $(\tilde{\pi}, C(T), T)$ satisfies our assertion.

q.e.d.

LEMMA 2. There exist normal irreducible complete hyperbolic com- 
plex spaces $\tilde{U}$ and $\tilde{V}$ with bimeromorphic mappings $s: \tilde{V} \rightarrow \tilde{U}$ and $t: \tilde{U}$ $\rightarrow \tilde{V}$, where $t$ is not holomorphic.

Proof. Take a triple system $(f, S, T)$ as in Lemma 1 . Let $\Gamma_{f}$ be the graph of the meromorphic mapping $f: S \rightarrow T$ and $\pi: \Gamma_{f} \rightarrow S$ the canonical projection. Let $g: S \rightarrow \Gamma_{f}$ be the inverse meromorphic mapping of the proper modification $\pi: \Gamma_{f} \rightarrow S$. Then there exists a point $x_{0}$ of $S$ such that $g\left(x_{0}\right)$ is not a single point, because $f$ is not holomorphic. Take an open neighborhood $\tilde{U}$ of $x_{0}$ in $S$ which is complete hyperbolic. Since $S$ is normal, we may assume that $\tilde{U}$ is also irreducible. Let $V=\pi^{-1}(\tilde{U})$ and $\mu: \tilde{V} \rightarrow V$ be a normalization of $V$. Being an analytic subset of the complete hyperbolic complex space $\tilde{U} \times T, V$ is also complete hyperbolic. Then, from a result of Kwack [4], $\tilde{V}$ is complete hyperbolic. Moreover, since $\tilde{U}$ is irreducible, so are $V$ and $\tilde{V}$. We now define meromorphic mappings $s: \tilde{V} \rightarrow \tilde{U}$ and $t: \tilde{U} \rightarrow \tilde{V}$ by $s=\psi \circ \mu$ and $t=\nu \circ \omega$, where $\psi: V \rightarrow \tilde{U}$ is the restriction of $\pi: \Gamma_{f} \rightarrow S$ to $V, \nu: V \rightarrow \tilde{V}$ is the inverse meromorphic mapping of the proper modification $\mu: \tilde{V} \rightarrow V$ and $\omega: \tilde{U} \rightarrow V$ is the restriction of $g: S \rightarrow \Gamma_{f}$ to $\tilde{U}$, respectively. Then we can show that $s: \tilde{V} \rightarrow \tilde{U}$ and $t: \tilde{U} \rightarrow \tilde{V}$ are bimeromorphic mappings and the inverse to each other. From our construction, it is clear that $t: \tilde{U} \rightarrow \tilde{V}$ is not holomorphic.

q.e.d.

Proof of Theorem 2. Let $\tilde{U}, \tilde{V}, s: \tilde{V} \rightarrow \tilde{U}$ and $t: \tilde{U} \rightarrow \tilde{V}$ be complex spaces and bimeromorphic mappings as in Lemma 2. Putting $X=\tilde{U}$ $\times \tilde{V}$, we define a bimeromorphic automorphism $\phi$ of $X$ by $\phi(u, v)$ $=(s(v), t(u))$ for $(u, v) \in X$. Then $X$ is a normal irreducible complete hyperbolic complex space. Moreover $\phi$ cannot be a biholomorphic automorphism of $X$. In fact, if it were so, both $s: \tilde{V} \rightarrow \tilde{U}$ and $t: \tilde{U} \rightarrow \tilde{V}$ are necessarily biholomorphic mappings. This contradicts the fact $t: \tilde{U} \rightarrow \tilde{V}$ is not holomorphic. Therefore we have shown that $\operatorname{Aut}(X)$ $\neq \operatorname{Bim}(X)$.

q.e.d.

\section{REFERENCES}

[1] H. Hironaka, Resolution of singularities of an algebraic variety over a field of characteristic zero, Ann. of Math. 79 (1964), 109-326.

[2] S. Kobayashi, Hyperbolic Manifolds and Holomorphic Mappings, Marcel Dekker, New York 1970.

[ 3 ] — Intrinsic distances, measures and geometric function theory, Bull. Amer. Math. Soc. 82 (1976), 357-416.

[4] M. H. Kwack, Generalization of the big Picard theorem, Ann. of Math. 90 (1969), 
9-22.

[ 5 ] H. Whitney, Complex Analytic Varieties, Addison-Wesley, Reading, Mass. 1973.

Akita University 